\title{
La competencia intercultural y el error pragmático
}

\author{
Intercultural Competence and Pragmatic Error
}

\author{
Cecylia Tatoj \\ Uniwersytet Śląski w Katowicach (Polonia) \\ cecylia.tatoj@us.edu.pl
}

\begin{abstract}
This paper discusses two topics which are very popular nowadays among the academics dealing with foreign language teaching, namely: intercultural competence and pragmatic error. The author notes the fact that those two notions appear separately, because the starting points for discussing them are usually different fields of science. The aim of the paper is to demonstrate that on one hand the two notions need to be treated uniformly, and on the other, that the pragmatic error should be included in error classifications which are references for both the authors of handbooks as well as people educating future teachers. For that purpose, the author defines intercultural competence, presents various approaches to error and its classification in teaching, and then explains what pragmatic error is and describes its linguistic characteristics.
\end{abstract}

Keywords: second language learning, error analysis, intercultural competence, pragmatics, pragmatic errors

\section{INTRODUCCIÓN}

En nuestro artículo tratamos dos temas actualmente muy en boga entre los investigadores que se ocupan de la enseñanza de lenguas extranjeras, a saber: la competencia intercultural y el error pragmático. Queremos llamar la atención de nuestros lectores acerca del hecho de que los dos conceptos suelen aparecer separadamente, ya que la competencia intercultural es abordada desde los puntos de vista sociológico, antropológico y didáctico, mientras que el error pragmático pertenece al campo de la lingüística. El objetivo de nuestro artículo se centrará, por un lado, en evidenciar que los dos conceptos deberían ser tratados como elementos continuamente interrelacionados y, 
por otro, en demostrar que el error pragmático debería ser incluido en las clasificaciones de errores que sirven de referencia tanto para los autores de manuales como para los futuros profesores de lenguas extranjeras.

Para alcanzar ambas finalidades a lo largo de nuestro artículo, comenzaremos con la definición de la competencia intercultural, así como con un acercamiento al concepto de error y su clasificación en la enseñanza de lenguas extranjeras. Posteriormente explicaremos qué es el error pragmático desde nuestro punto de vista y describiremos sus características, apoyándonos en las aportaciones provenientes de la lingüística. Para finalizar, añadimos algunos ejemplos basados en estudiantes españoles que han participado en el programa Erasmus+ en la Universidad de Silesia.

\section{LA COMPETENCIA INTERCULTURAL}

Para poder definir la competencia intercultural, comenzaremos con la definición de cultura, lo cual no es nada fácil. Seguiremos a Hall (1989, p. 16), quien percibe la cultura como un conjunto de códigos y añade que sin consideración al número de diferencias que se refieren a los detalles, los antropólogos están de acuerdo en que la cultura tiene tres características: (1) no es innata, sino aprendida; (2) sus partes están interiormente trabadas, es decir, si abordamos uno de sus aspectos, se tocan todos; y (3) es una característica común a todos los hombres pero que marca las fronteras entre diferentes grupos sociales.

Podemos decir, pues, que la cultura está estrictamente ligada al comportamiento humano y a las expectativas que tenemos hacia nuestros interlocutores. Por lo tanto, la competencia intercultural se puede definir como el conocimiento de las diferencias entre culturas que influye en el cambio de nuestras expectativas comunicativas o, más bien, nos hace conscientes de ellas y facilita que las modifiquemos.

Żydek-Bednarczuk (2015, pp. 76-77) presenta un catálogo de bases relacionadas con la competencia intercultural. Así, afirma que la competencia intercultural:

1. Depende de cada persona y se realiza en situaciones concretas. Puede ser diferente para cada uno, según sus necesidades, objetivos y motivaciones.

2. Es muy difícil medirla. Más bien hablamos de las impresiones que tienen los interlocutores en el proceso de comunicación intercultural.

3. Es gradual. Depende del grado de conocimiento, motivación y capacidad.

4. No puede dominarse de forma ideal, ya que las culturas, actividades y situaciones cambian (Grucza, 2000, p. 28, citado por Żydek-Bednarczuk, 2015).

5. Se aprende en cada grado del conocimiento lingüístico.

6. La diferencia cultural provoca diversos comportamientos: adquisición de la competencia, aprendizaje reproductivo de los comportamientos culturales o desapego hacia una cultura diferente y la preferencia por la propia cultura ${ }^{1}$.

\footnotetext{
${ }^{1}$ Todas las traducciones del polaco son nuestras.
} 
En cuanto a la didáctica de las lenguas extranjeras, manifestamos nuestro acuerdo con Spychała (2010, p. 81), según la cual "[...] es imprescindible conectar la enseñanza de la cultura con la lengua, cosa que cada vez más parece ser obvia para los profesores de ELE" y con Ellis (2004, p. 542), que subraya que la disposición comunicativa no es una herramienta para mejorar la competencia comunicativa, sino el objetivo final en la enseñanza de lenguas extranjeras. Sin embargo, postulamos que la disposición comunicativa ha de entenderse como la disposición de la comunicación intercultural.

Además, queremos subrayar que el concepto de la competencia intercultural se debería tratar como un concepto difuso que se puede ver desde distintas perspectivas. La que proponemos es la lingüística, porque en la mayoría de los casos el comportamiento humano está estrictamente unido a la lengua, pues hay muy pocas situaciones interpersonales en las que no hablemos. Al mismo tiempo, la lengua está estrechamente ligada a la cultura: nos comportamos siguiendo unas normas sociales que hemos aprendido de forma activa y no consciente. De todo esto podemos deducir que la competencia intercultural está ligada a las diferencias pragmáticas entre hablantes que provienen de distintas culturas, es decir, que: "se entiende la habilidad del aprendiente de una segunda lengua o lengua extranjera para desenvolverse adecuada y satisfactoriamente en las situaciones de comunicación intercultural que se producen con frecuencia en la sociedad actual, caracterizada por la pluriculturalidad" (Centro Virtual Cervantes, Competencia intercultural). En consecuencia, la carencia de competencia intercultural nos lleva directamente al concepto de error pragmático. Sin embargo, antes de acercarnos al mismo, nos gustaría tratar brevemente el tema de la clasificación de los errores.

\section{DEFINICIÓN Y CLASIFICACIÓN DE LOS ERRORES EN LA ENSEÑANZA DE LENGUAS EXTRANJERAS}

En la enseñanza de las lenguas extranjeras, por un lado hablamos de las competencias que tienen que aprender los alumnos (como la competencia intercultural) y, por otro, de los errores que cometen. Estos últimos, siguiendo a Corder (1983, p. 117), vamos a entenderlos como la violación del código, es decir, el uso de reglas inadecuadas o el uso inadecuado de las reglas adecuadas. Sin embargo, los investigadores se preguntan qué criterios hay que contemplar al describirlos². Según Łukasik (2017, pp. 257-258), si queremos averiguar si se ha cometido un error o no, hay que determinar la evaluación desde distintos puntos de vista: la gramaticalidad, la aceptabilidad y la comunicación. En el último caso, lo que nos interesa es determinar si el error que se ha cometido influye en la comunicación o no; podemos decir, de hecho, que está unido a la aceptabilidad.

\footnotetext{
${ }^{2}$ Para más cuestiones sobre el análisis y corrección de errores, véase Muños-Basols \& Bailini (2018).
} 
Por otro lado, la crítica no siempre está de acuerdo en la enumeración de los tipos de los errores. Por ejemplo, Markowski (2002) propone la división de los errores lingüísticos en extra- e interlinguales. Los primeros son errores ortográficos y de puntuación; los segundos, errores de sistema (lingüísticos) y errores de uso (estilísticos): entre los lingüísticos encontramos los gramaticales (de flexión y de sintaxis), los léxicos (de léxico, fraseología y formación de palabras) y los fonéticos. Por otra parte, Grucza (1978, p. 60) habla de errores de pronunciación, gramaticales, de léxico y de fraseología. Otra clasificación viene de la mano de Vázquez (1999, p. 28), que distingue entre errores lingüísticos, etiológicos y comunicativos. No encontramos obras dedicadas a la clasificación de errores en los que se hable directamente de errores pragmáticos; sin embargo, hay muchos trabajos dedicados a la competencia pragmática en los que se habla de este tipo de errores (véase por ejemplo: Blum-Kulka, 1989; Escandell, 1996; Gómez Marón, 2004; Bravo \& Briz, 2004; Barquero Pipín, 2020).

Al mismo tiempo, es muy importante añadir que hay varias disciplinas que se interesan por el error, sobre todo la lingüística y la didáctica, poseyendo cada una de ellas su propia perspectiva ${ }^{3}$. Si tratamos la lengua solamente desde el punto de vista sintáctico, semántico y fonético, la perspectiva parece convergente: tanto a los lingüistas como a los profesores, nos interesa que el alumno no cometa errores que puedan afectar a la comunicación. Sin embargo, si añadimos la perspectiva pragmática, el enfoque cambia drásticamente: lo que para el lingüista constituye una violación de las máximas conversacionales formuladas por Grice (1975) que puede llevar directamente a un malentendido, desde el punto de vista didáctico, no tiene por qué ser así, sobre todo si hablamos de un profesor no nativo, que se desenvuelve en el mismo nivel cultural que el alumno. En este caso, un severo error pragmático puede permanecer invisible para este. Así que es relativamente fácil destacar, clasificar y evaluar los errores en el plano formal de la lengua, hecho imposible con los errores pragmáticos que, como parte del comportamiento humano, dejan un gran margen a la subjetividad.

\section{LAS CARACTERÍSTICAS DEL ERROR PRAGMÁTICO}

Como subraya Sztuk (2017, p. 15), "[d]esde el punto de vista lingüístico, el objetivo primordial de la actividad humana es la comunicación y, por tanto, en pocas palabras, la comunicación transmitiendo y recibiendo las intenciones de otros participantes de la situación comunicativa. Mirando a través del prisma de la pragmática, lograremos este objetivo cuando, por un lado, nuestro discurso llegue al destinatario y evoque una respuesta adecuada, y cuando recibamos y comprendamos el mensaje de retroalimentación, y con él la intención del emisor". Partiendo de esta base, optamos por unir el con-

\footnotetext{
${ }^{3}$ Por ejemplo, Kwapisz-Osadnik (2018) trata la cuestión sobre el error lingüístico al servicio de una enseñanza de idiomas eficaz.
} 
cepto de competencia intercultural con la pragmática, ya que la falta de la competencia intercultural provoca errores pragmáticos entre representantes de distintas culturas.

Aunque en numerosas ocasiones nos parece que las normas de comportamiento aceptadas por diversas culturas deberían ser objetivas, propias de toda la humanidad, no es así. La percepción de una situación concreta no es ni lógica ni objetiva y puede variar en culturas concretas, ya que se trata de un conocimiento de normas implícitas que se aprenden en esa sociedad, pero que percibimos como universales debido a la visión etnocéntrica, propia de la mayoría de los miembros de dicha sociedad. Esta visión explica por qué la competencia intercultural no está sujeta al principio de progresión cíclica, es decir, por qué los alumnos no aprenden a autocorregir los errores pragmáticos en los niveles más altos de dominio de una lengua, aunque sí que son capaces de corregirse en los niveles semántico, sintáctico y fonético. Como escriben Nęcka, Orzechowski \& Szymura (2007, p. 25), "el supuesto básico con respecto a los procesos de procesamiento de información es el supuesto sobre la economía del discurso. De acuerdo con este supuesto, procesar toda la información disponible no tendría sentido, la mayor parte sigue siendo inútil desde el punto de vista de los requisitos de la situación en la que se encuentra el organismo". Según Fiske y Taylor (1991), el hombre es un avaro cognitivo, es decir, una criatura que normalmente utiliza solo una parte de los recursos cognitivos que tiene disponibles. Desde esta premisa podemos entender los errores pragmáticos: una vez aprendidas las reglas de la vida social, el alumno no las revisa, o sea, aunque hable otra lengua, sigue comportándose como lo hace cuando usa su lengua materna.

Y aunque, como dice Fernández (1997, p. 16), "numerosas investigaciones han puesto de manifiesto que los errores debidos a interferencias de la LM alcanzan como máximo un promedio del 33\% del total de los errores producidos por los aprendices", apreciamos, según nuestras investigaciones, que los errores pragmáticos son diferentes. Como ya hemos señalado, el alumno que usa una lengua extranjera sigue comportándose igual, culturalmente hablando, así que la interferencia de la cultura materna es muy grande. Esto explica por qué comenten también estos errores personas con un alto dominio de una LE.

Simultáneamente, la falta de competencia intercultural no se tolera tan fácilmente como la de competencia lingüística. Como subraya Portolés (2004, p. 25), una persona que no domina las reglas pragmáticas puede ser considerada maleducada. Nuestra experiencia con los alumnos de intercambio Erasmus muestra que las personas de culturas diferentes bien acusan de falta de cortesía, bien opinan que los otros se comportan de una forma exageradamente culta, lo que tampoco es deseable. Así, a partir de esta aseveración vale la pena subrayar otra característica del error pragmático: puede ser bilateral, es decir, puede que los dos interlocutores se acusen uno al otro, de un comportamiento raro, inadecuado en una situación concreta.

Paralelamente, los errores pragmáticos están sobre todo ligados a la función social del lenguaje: se manifiestan cuando usamos la lengua para mantener relaciones socia- 
les y, en menor medida, cuando lo hacemos para transmitir una información concreta. Al respecto, hemos de mencionar los resultados de una investigación realizada por Emler (1994, citado por Gazzaniga, 2013), el cual señala que en aproximadamente el $80-90 \%$ de todas las conversaciones, aparecen nombres específicos y personas conocidas. Esto implica que la mayor parte de las interacciones se realizan para mantener contactos. Como subraya Gazzaniga (2013, p. 136), la mayoría de nuestras conversaciones son cotilleos, lo que significa que somos entes completamente sociales. Esto conlleva que, aunque poco visibles, los errores pragmáticos parecen ser los mayores responsables de los malentendidos. Al hablar, por un lado transmitimos una información concreta, pero por otro, en la mayoría de casos, creamos relaciones sociales. Para que una persona nos acepte, la imagen de nosotros mismos que le transmitimos tiene que ser agradable, amistosa $\mathrm{y}$, sobre todo, coherente ${ }^{4}$.

El error pragmático surge de la falta de conocimiento de las reglas que rigen situaciones sociales concretas, pues es necesario darse cuenta de que, como subraya Rothenbuhler (1998), toda nuestra vida social está ritualizada, es decir, en las relaciones sociales usamos unas normas concretas que al mismo tiempo no existen sin nuestra aceptación. Las reglas por un lado nos limitan, pero por otro dan una seguridad, sobre todo porque nos dicen cómo comportarnos en una situación concreta y en función de las mismas podemos prever cómo se van a comportar nuestros interlocutores: o sea, en relación con estas conformamos unas expectativas hacia otras personas. Consideramos que el error pragmático está unido con esas expectativas hacia el interlocutor: sin darnos cuenta, suponemos cómo alguien debería comportarse en una situación dada, cómo debería responder, incluso, qué palabras debería usar, es decir, sin percatarnos establecemos un sesgo determinado. Si analizamos el comportamiento humano, se hace necesario citar a Gazzaniga (2013, p. 125), según el cual "la capacidad de juzgar a otras personas es esencial para navegar de manera eficiente por el mundo social”.

En contraste con otras normas, las normas sociales no son tan evidentes, tan visibles ni tampoco son explícitas (aunque algunas sí que se pueden encontrar en los libros dedicados a la urbanidad y buenos modales) aunque, sobre todo, tampoco son tan estudiadas y explicadas en las clases de idiomas. La invisibilidad de los errores pragmáticos nos explica también por qué no se suelen enseñar de forma teórica y regular. Como subraya Robert (2008), incluso una revisión superficial de los libros de texto de lenguas extranjeras muestra que las competencias generales, principalmente socioculturales, están insuficientemente desarrolladas.

Nos damos cuenta de que es difícil enseñar la competencia intercultural, por lo que, según nuestra opinión, esta habría de enfocarse en la sensibilización del alumno hacia las reglas de la vida social que varían en distintos ámbitos, es decir, en "sectores amplios de la vida social en los que actúan los agentes sociales” (MCER, 2002, p. 10).

\footnotetext{
${ }^{4}$ Para ampliar el tema véase, por ejemplo, Calsamiglia \& Tusón (2012, pp. 156-163) o Nowakowska-Głuszak (2009), así como el trabajo sobre el ethos comunicativo escrito por Baran (2010).
} 
En otras palabras, el aprendiz tiene que entender que la vida es, como ya subrayaba el sociólogo Goffman (1959), un teatro en el que el hombre interpreta varios papeles; en el caso de nuestros alumnos son papeles tales como alumno, hijo, nieto o cliente, y en todas las situaciones tiene que comportarse de una manera adecuada. Este comportamiento le ofrece seguridad, aunque también muestra a sus interlocutores que es una persona conocedora de los códigos culturales y de las normas sociales y, además, los cumple manteniendo de este modo el orden social. Sin embargo, el alumno tiene que saber que "la manera adecuada" puede entenderse de distintas formas. Se le debería explicar que las normas sociales que ha aprendido en su ámbito vernáculo parecen "naturales" y "normales", mientras que las otras son entendidas como "ajenas"; así que necesita comprender el funcionamiento de las nuevas normas sociales y aprender cómo interactuar con unas normas diferentes a las que ha aprendido desde pequeño. De esta manera, le enseñamos la mediación intercultural, la empatía hacia los representantes de otras culturas, e incluso podemos reflexionar acerca de cómo se forman los estereotipos nacionales ${ }^{5}$.

Como botón de muestra acerca de lo dicho, nos gustaría comentar brevemente algunos resultados provenientes de nuestras investigaciones llevadas a cabo en la Universidad de Silesia en el periodo comprendido entre los años 2002-2020, las cuales han tenido como sujetos de estudio a los estudiantes españoles que han participado en el programa Erasmus en esta universidad. De esta forma, hemos constatado que la mayoría de malentendidos que han tenido lugar están relacionados con una visión diferente de los roles del estudiante universitario que tienen los alumnos españoles y los profesores polacos. Las interacciones entre un estudiante y su profesor en el ámbito universitario español parecen ser más libres y abiertas. Sin embargo, en Polonia se exige que el alumno, al interactuar con el profesor no solamente le trate de usted, sino que también sepa qué título académico posee y lo use durante la interacción oral y, especialmente, durante la interacción escrita. Así, por ejemplo, a un doctor, el alumno lo trata como "Panie Doktorze" [Señor Doctor], y al inicio de un texto escrito le saluda del siguiente modo: "Szanowny Panie Doktorze!" [Estimado Señor Doctor].

Al mismo tiempo, cuando se interactúa con los profesores, los alumnos polacos mantienen una mayor distancia, esperan su turno de palabra sin interrumpir al profesor $y$, de vez en cuando, inclinan la cabeza, indicando comprensión y respeto ${ }^{6}$. Cuando piden algo al profesor, suelen usar el condicional simple: "Czy mógłby Pan" [Podría usted]. Asimismo, si asisten a una tutoría individual, no suelen hablar mucho $\mathrm{y}$, tras finalizar el tema abordado por el profesor, salen directamente de su despacho.

La otra cara de la moneda viene dada por los estudiantes españoles, debido a que desconocen las reglas que rigen la vida universitaria polaca, por lo cual se comportan

\footnotetext{
${ }^{5}$ Para desarrollar el tema véase, por ejemplo, Tatoj (2019).

${ }^{6}$ Lógicamente, en cualquier interacción comunicativa la proxemia desempeña un papel importante, tanto en las matizaciones complementarias al discurso, como en las formulaciones quinésicas destinadas a manifestar rasgos de cortesía o de descortesía.
} 
como si se hallaran en una universidad española: suelen tutear al profesor, no mantienen las distancias y al interactuar con él suelen interrumpirle. Por otra parte, cuando le solicitan algo suelen usar el modo imperativo ${ }^{7} \mathrm{y}$, si se trata de la asistencia a una tutoría individual, suelen hablar bastante, pasando en el despacho del profesor un tiempo prolongado antes de salir del mismo, aunque la tutoría ya haya finalizado con anterioridad, habida cuenta de que corresponde al profesor finalizar la conversación.

De esta forma, los profesores polacos tienen la percepción acerca de los estudiantes españoles de ser personas que vulneran determinadas normas sociales, ser demasiado directas, e incluso, a veces, maleducadas. Por otro lado, si explicamos a los estudiantes españoles las reglas culturales polacas, estos las perciben como un hecho en exceso artificial. En este caso, podemos decir que ambos grupos se catalogan recíprocamente como "extraños", dado que entendemos que las normas sociales que se han aprendido en su entorno natal parecen "naturales" y "normales", mientras que las otras, son etiquetadas como "raras". Por todo ello hemos de concluir indicando que nuestros alumnos necesitan entender el funcionamiento de las normas sociales y aprender cómo interactuar con unas normas diferentes a las que han adquirido.

Una vez señalados estos ejemplos, consideramos imprescindible enseñar la competencia intercultural basándonos en la definición del error pragmático. Nos parece muy importante unir los dos conceptos, ya que la comprensión de las características del error pragmático lleva directamente al entendimiento del funcionamiento de la competencia intercultural y a su gradual mejora.

\section{A MODO DE CONCLUSIÓN}

Tal como hemos ido planteando la cuestión en este artículo, hoy en día se habla mucho tanto sobre la competencia intercultural como sobre los errores pragmáticos, pero como ambos conceptos suelen ser tratados desde distintos puntos de vista, provenientes de diferentes campos de investigación, no son abordados de forma unitaria en la clase de lenguas extranjeras. Nosotros, sin embargo, postulamos hacerlo, ya que, según nuestra opinión, en la interculturalidad el punto de vista lingüístico puede ayudar a los profesores a entender el funcionamiento de los errores pragmáticos de los estudiantes y, en consecuencia, enseñarles a corregirlos.

Vemos muy oportuno añadir los errores pragmáticos en los análisis de errores. El hecho de demostrar que son errores muy particulares, pero que paralelamente influyen sobremanera en la comunicación, nos hace colegir la imprescindibilidad de incluirlos en las clasificaciones de errores para que sirvan de referencia especialmente a quienes se ocupan de la enseñanza a los futuros profesores.

\footnotetext{
${ }^{7}$ Para una comparación de las peticiones en español y polaco, véase Nowakowska-Głuszak (2009).
} 


\section{BIBLIOGRAFÍA}

Baran, M. (2010). Emotividad y convención sociopragmática: una contribución al estudio del ethos comunicativo de la comunidad hispanohablante peninsular. Łódź: Wydawnictwo Uniwersytetu Łódzkiego.

Barquero Pipín, A.C. (2020). Lengua, cultura, interculturalidad. El tratamiento de la competencia pragmática como parte de la competencia comunicativa en los libros de texto de ELE en el ámbito escolar alemán. Potsdam: Universitätverlag Potsdam.

Blum-Kulka, S., House, J., Kasper, G. (Eds.) (1989). Cross-Cultural Pragmatics: Request and Apologies. Norwood (NJ): Ablex.

Bravo, D., Briz, A. (Eds.) (2004). Pragmática sociocultural: estudios sobre el discurso de cortesía en español. Barcelona: Ariel.

Calsamiglia, H., Tusón, A. (2012). Las cosas del decir. Manual de análisis del discurso. Barcelona: Ariel.

Centro Virtual Cervantes, Competencia intercultural. Recuperado de: https://cvc.cervantes.es/ensenanza/ biblioteca_ele/diccio_ele/diccionario/compintercult.htm.

Consejo de Europa (2002). Marco Común Europeo de referencias para las lenguas: aprendizaje, enseñan$z a$, evaluación. Madrid: Anaya.

Corder, S.P. (1983). Analiza błędu językowego. In J.P. Brierley Allen, S.P. Corder, \& J. Rusiecki (Eds.), Kurs edynburski językoznawstwa stosowanego, t. II: Techniki w językoznawstwie stosowanym (pp. 116-131). Warszawa: Wydawnictwa Szkolne i Pedagogiczne.

Ellis, R. (2004). Individual Differences in Second Language Learning. In A. Davies, C. Elder (Eds.), The Handbook of Applied Linguistics (pp. 525-551). Oxford: Blackwell.

Escandell-Vidal, M. (1996). Los fenómenos de interferencia pragmática. In Didáctica del español como lengua extranjera (pp. 95-110). Madrid: Fundación Actilibre.

Fernández, S. (1997). Interlengua y análisis de errores en el aprendizaje del español como lengua extranjera. Madrid: Edelsa.

Fiske, S.T., Taylor, S.E. (1991). Social cognition. New York: McGraw-Hill.

Gazzaniga, M.S. (2013). Kto tu rządzi - ja czy mój mózg? Neuronauka a istnienie wolnej woli. Sopot: Smak Słowa.

Goffman, E. (1959). The Presentation of Self in Everyday Life. Garden City, New York: Doubleday.

Gómez Marón, R. (2004). La competencia pragmático-conversacional en los manuales de español como lengua extranjera: breve revisión teórico-práctica desde la teoría pragmática de la cortesía lingüística. In F.L. Bergillos \& S. Ruhstaller (Eds.), La competencia lingüistica y comunicativa en el aprendizaje del español como lengua extranjera (pp. 141-154). Madrid: Edinumen/Universidad Pablo de Olavide.

Grice, P. (1975). Lógica y Conversación. In L. Valdés (Ed.), La búsqueda del significado (pp. 511-530). Madrid: Tecnos/Universidad de Murcia.

Grucza, F. (Ed.) (1978). Z problematyki błędów obcojęzycznych. Warszawa: Wydawnictwa Szkolne i Pedagogiczne.

Hall, E.T. (1989). Beyond culture. New York: Anchor Books.

Kwapisz-Osadnik, K. (2018). L'erreur linguistique au service de l'enseignement efficace des langues. Synergies Pays Riverains de la Baltique, 12, 65-74.

Łukasik, M. (2017). Słownik wyrażeń metanaukowych, metalingwistycznych i metakulturologicznych Profesora Franciszka Gruczy. In S. Grucza (Ed.), Franciszek Grucza. Dzieła zebrane, t. 1: O nauce prof. Franciszka Gruczy (pp. 252-348). Warszawa: Wydawnictwo IKSI.

Markowski, A. (2002). Wielki stownik poprawnej polszczyzny. Warszawa: PWN.

Muñoz-Basols, J., Bailini, S. (2018). Análisis y corrección de errores. In J. Muñoz-Basols, E. Gironzetti \& M. Lacorte (Eds.), The Routledge Handbook of Spanish Language Teaching. Metodologias, contextos y recursos para la enseñanza del español L2 (pp. 94-108). London-New York: Routledge. 
Nęcka, E., Orzechowski, J., Szymura, B. (2007). Psychologia poznawcza. Warszawa: PWN.

Nowakowska-Głuszak, A. (2009). ¿Pedir es también cuestión de cultura?: análisis comparativo de las peticiones en la lengua española y polaca desde el punto de vista de la lingüística cultural. Katowice: Wydawnictwo Uniwersytetu Śląskiego.

Portolés, J. (2004). Pragmática para hispanistas. Madrid: Síntesis.

Robert, J.-P. (2008). Dictionnaire pratique de didactique du FLE. Paris: Ophrys.

Rothenbuhler, E.W. (1998). Ritual communication: from everyday conversation to mediated ceremony. Thousand Oaks: Sage Publications.

Spychała, M. (2010). El enfoque cultural en la enseñanza y el aprendizaje de español como lengua extranjera - ELE. Studia Romanica Posnaniensia, 37 (2), 71-83. DOI: 10.14746/strop.2010.372.007.

Sztuk, A. (2017). Normalizacja translatoryczna. Warszawa: Wydawnictwo Naukowe Instytutu Komunikacji Specjalistycznej i Interkulturowej, Uniwersytet Warszawski.

Tatoj, C. (2019). ¿Qué esconden las palabras? La enseñanza del léxico y los estereotipos nacionales. Verba Hispanica, 27 (1), 11-27. DOI: 10.4312/vh.27.1.11-27.

Vázquez, G. (1999). ¿Errores? ¡Sin falta! Madrid: Edelsa.

Żydek-Bednarczuk, U. (2015). Spotkanie kultur: komunikacja i edukacja międzykulturowa w glottodydaktyce. Katowice: Wydawnictwo Uniwersytetu Śląskiego. 